Radar came out of the war with a glory that gave a false impression of the ease with which it could be adapted to peaceful purposes. This adaptation involved considerable research by manufacturers into the techniques of radar, and by users into ways of using it at sea. It was the users who first achieved success by having a code of radar conduct agreed. This done they can maintain that the manufacturers have not yet given them the means to put the code into proper operation.

Having already seen in the realm of radar use and traffic regulation at sea, how true it is that unity is strength, mariners are now in a position to say to the manufacturers: 'You have already offered us all sorts of good things, but to satisfy us completely you should give us compass-rose presentation and automatic recording'.

\title{
REFERENCES
}

1 Sadler, D. H. (1957). The mathematics of collision avoidance at sea, this Journal, 10, 306 .

2 The value of a mathematical approach to the collision problem, this Journal, 16, $189(1963)$.

3 Wepster, A. (1962). The arrangement of navigational equipment in modern cargo vessels, this Journal, 15, 241.

\section{The Sun-Star Chronometer}

from Air Marshal Sir Edward Chilton, K.B.E., C.B.

THE Sun-Star Chronometer described in this note has been developed by Raymond Nardin of Switzerland and Oscar E. Batori of the United States; it is manufactured by Ulysse Nardin and produced by the Batori Computer Company Inc. of New York. It contains interesting features which merit description.

The Chronometer has two movements of different speeds, one corresponding to solar time and the other to sidereal time, connected by a planetary differential mechanism and regulated by a single system, so that the two movements have a common rate and can be stopped and started simultaneously. All the hands (including the second hands) can be set and a single correction can be applied for either solar or sidereal time.

The Chronometer, which indicates simultaneously, solar and sidereal time, is designed primarily for navigation by the stars: G.H.A. Aries is available on inspection, as Mean Time is from an ordinary watch. In the illustration the time is shown as G.M.T. $15^{\mathrm{h}} 2 \mathrm{I}^{\mathrm{m}} 28^{\mathrm{s}}$ and G.H.A. Aries (or Sun) $49^{\circ} 03^{\prime} 50^{\prime \prime}$. The hands for time and arc are differentiated by colour.

G.H.A. Sun can be obtained by making a correction of $2: 5$ per hour over short periods, or 59:1 per day and an average equation of time for longer periods. These corrections can be precalculated. 


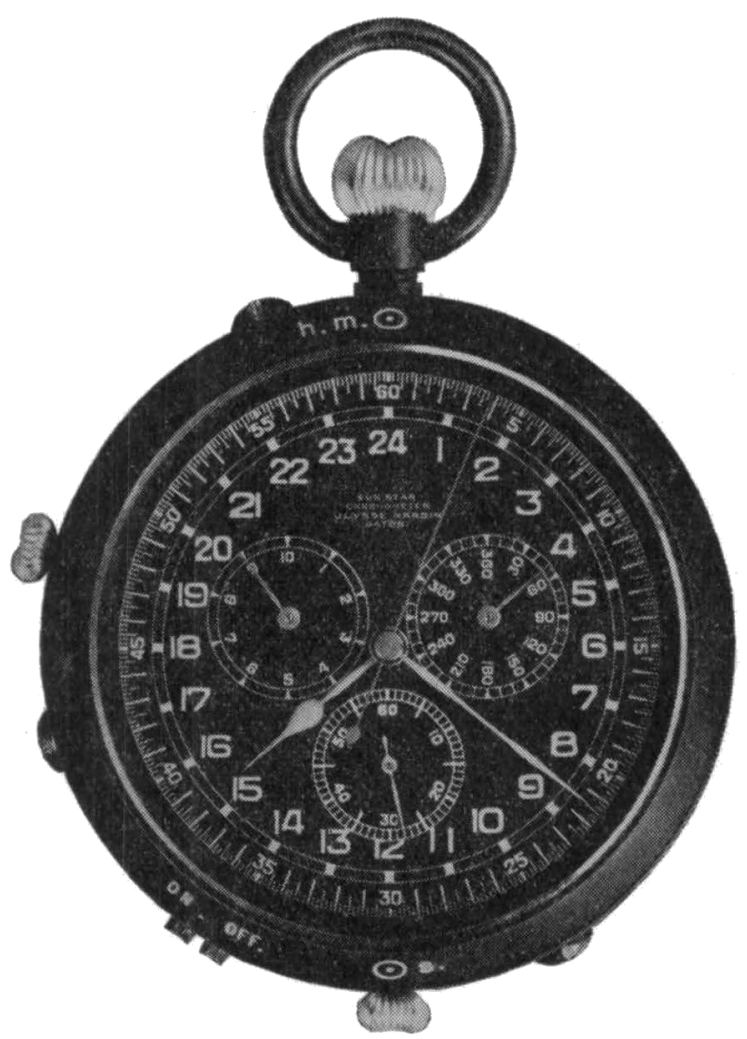

FIG. I. The Sun-Star Chronometer

The Chronometer has been extensively tested on the ground and in the air and has passed the Royal Greenwich Observatory accuracy tests. A report from the Ministry of Aviation test establishment at Boscombe Down states: 'The Chronometer was found useful when sighting celestial bodies on an opportunity basis, when up to 20 seconds in time was saved in obtaining L.H.A.; a process which takes about 30 seconds using the Air Almanac.' The same source states: 'Current astro-techniques using the periscopic sextant require altitude and azimuth to be precomputed in order to set these values initially on the sextant. When the Chronometer was used to assist in precomputation, increments of G.H.A. had to be applied to the Chronometer reading in order to obtain the G.H.A. of Aries for the time of the astro shot. Used in this way, the Chronometer offered little advantage over the Air Almanac.' On the other hand conditions are not always favourable for precomputation and the Sun-Star Chronometer offers a flexible method which is independent of date and time and eliminates all interpolation. 